\title{
Presentación de una escala para evaluar actitudes y creencias sobre la sexualidad reproductiva en adolescentes varones de la región Caribe colombiana*
}

Presentation of an Attitudes and Beliefs Scale to

Assess Adolescent Sexuality and Reproductive Health of Young Males in the Caribbean Region

Recibido: junio 10 de 2012 | Revisado: marzo 1 de 2013 | Aceptado: marzo 26 de 2013

doi:10.11144/Javeriana.UPSY13-1.peea.

Para citar este artículo: Bello-Villanueva, A. M. Palacio, J., Vera-Villarroel, P., Oviedo-Trespalacios, O., Rodríguez-Díaz, M., Celis-Atenas, K., et al. (2014). Presentación de una escala para evaluar actitudes y creencias sobre la sexualidad reproductiva en adolescentes varones de la Región Caribe colombiana. Universitas Psychologica, 13(1), 47-60. doi:10.11144/Javeriana.UPSY13-1.peea.

* Esta investigación ha sido realizada como parte del proyecto financiado por Colciencias No 121549326168, titulado: "Validación de Estrategias Pedagógicas y Comunicacionales Mediante un Modelo Cognitivo Social y Epidemiológico para la Prevención de Comportamientos Sexuales de Riesgo, en Adolescentes Escolarizados entre 11-16 años de Dos Ciudades del Caribe Colombiano" y con el apoyo de la DIP, Universidad del Norte, Barranquilla-Colombia.

Agradecimientos: La autora principal desea agradecer el apoyo en la construcción de las Escalas de Actitud y de Creencias Normativas a la Dra. Elvia Vargas Trujillo, tutora en pasantía del Grupo Familia y Sexualidad, Departamento de Psicología, Facultad de Ciencias Sociales, Universidad de los Andes, Colombia, también agradecer al proyecto USA 1204 del Ministerio de Educación/CITIAPS.

$* *$ Correspondencia: Ana Mercedes Bello Villanueva. Universidad del Norte. A.A. 1569-15820 Barranquilla-Atlántico, Colombia.E-mail: bellom@uninorte.edu.co

**** Grupo de investigaciones en Desarrollo Humano (GIDHUM), Departamento de Psicología, Universidad del Norte, Barranquilla, Colombia.

****** Escuela de Psicología, Universidad de Santiago de Chile (USACH), Santiago de Chile.

**:**** Departamento de Ingeniería Industrial, Barranquilla, Universidad del Norte, Colombia.

\author{
Ana Mercedes Bello-VillanueVA ** \\ Oscar OviEdo-Trespalacios $* * *$ \\ Universidad del Norte, Colombia \\ PABlO Vera-VILLARROEL ***** \\ Universidad de Santiago de Chile, Chile \\ ÓsCAR OVIEDo***** \\ Melissa RODRIGUEZ-DiaZ \\ Universidad del Norte \\ KAREM CELIS-ATENAS \\ Paula Pavez \\ Universidad de Santiago de Chile, Chile
}

\begin{abstract}
RESUMEN
Este artículo presenta la construcción y comprobación de la confiabilidad de un instrumento para evaluar las actitudes y normas subjetivas en el comportamiento sexual y reproductivo en varones en dos estudios. La escala fue administrada a 965 estudiantes varones escolarizados, entre 11 a 19 años de edad, en la región Caribe colombiana. En el Estudio 1 (pilotaje) el cuestionario constituido por 150 ítems se redujo a 78 en la prueba final. En la escala de actitud protección (Media $=4.1$ y $D E=1.07$ ) se obtuvo un alfa de Cronbach de 0.93 y en la de actitud abstinencia/retardo (Media $=$ 3.6 y $D E=1.26$ ), un alfa de 0.88 y homogeneidad elevada con correlación ítem-total superiores a 0.6. Para la escala de creencias categorizada como normas subjetivas (Media $=3.79$ y DE $=1.08$ ) la estimación del alfa de Cronbach fue de 0.98 y homogeneidad adecuada con correlaciones superiores a 0.66. La evaluación de la validez de constructo mediante el análisis factorial exploratorio y análisis de componentes principales con rotación VARIMAX, denota adecuación satisfactoria de los datos a la matriz factorial en el Estudio 2 (escala definitiva). En conclusión el instrumento es confiable, de estructura estable para la medición de actitudes y normas subjetivas en sexualidad reproductiva y contribuirá a establecer acciones específicas en programas, acorde a las expectativas en salud sexual y reproductiva de los adolescentes varones.

Palabras clave autores

Creencias, actitudes, sexualidad, reproducción, adolescentes varones.

Palabras clave descriptores

Pruebas psicológicas, psicometría, validación y confiabilidad.
\end{abstract}


Ana Mercedes Bello-Villanueva, Jorge Palacio, Pablo Vera-Villarroel, Oscar Oviedo-Trespalacios, Melissa Rodríguez-Diaz, Karen Celis-Atenas, Paula Pavez.

\section{A B S T R A C T}

This paper presents the construction and testing of the reliability of an instrument to assess attitudes and subjective norms sexual and reproductive behavior of young males. The scale was administered to 965 male students (From of 11 to 19 years) enrolled at school in the Colombian Caribbean region. This work includes two independent studies, in the Study 1 (pilot) the questionnaire consisted of 150 items and it was reduced to 78 . In the protective attitude scale (Mean $=4.1$ and $S D=1.07$ ) was obtained Cronbach's alpha of 0.93 and for the abstinence / delay attitude (Mean $=3.6$ and SD $=1.26$ ) an alpha of 0.88 with high homogeneity. The total correlation item was greater than 0.6 for all the attitude scales. On the scale of beliefs represented by the subjective norms (Mean $=3.79$ and $S D=1.08$ ) the estimation of Cronbach's alpha was 0.98 with correlations above 0.66 . In Study 2 (final stop), the construct validity assessment using factorial analysis and principal components analysis with VARIMAX rotation reported a satisfactory fit of the data to the array factor. In conclusion, the instrument has a reliable and stable structure for the measurement of attitudes and subjective norms for sexuality and reproductive health, and it is useful to help in the setting of programs.

Keywords authors

Beliefs, attitudes, sexuality, reproductive, young males.

Keywordss plus

Psychological test, psychometry, validation and fiability.

\section{Introducción}

En la actividad sexual adolescencial se ha explorado la relación existente entre las actitudes y el comportamiento sexual de los jóvenes y se ha encontrado que las actitudes personales de los adolescentes, junto con la percepción de estas en personas cercanas y significativas hacia el inicio de la actividad sexual, influyen sobre la disposición a tener relaciones sexuales en la adolescencia (Bearman \& Brückner, 1999; Carvajal et al., 1999; Ramiro, Bermúdez, Sierra \& Buela-Casal, 2013; Whitaker, Miller \& Clark, 2000).

Tanto Di Clement, Crosby y Wingood (2002) como Romer, Stanton, Galbraith, Feigelman, Black y Li (1999) plantean que las actitudes y creencias se elaboran y mantienen en la familia, en los grupos de pares, en los de referencia, en la sociedad y la cultura, e influyen en la prevención de comportamientos de riesgos sexuales en los adolescentes. Estas actitudes y creencias hacen parte de las estructuras cognitivas que las personas aprenden para organizar y sistematizar sus experiencias y su conducta. Por ejemplo, ciertos estudios señalan que las actitudes y creencias que asumen los adolescentes hacia la sexualidad se constituyen en un factor importante para la predicción de estas conductas sexuales (Jiménez, Mosquera \& Obregón, 2004; Lameiras, Rodríguez, Calado \& González, 2004; Mosquera, Jiménez \& Sanjuan, 2004).

Las creencias basadas en la influencia social afectan el mantenimiento de la salud y explican la relación entre emoción y salud, a su vez desempeñan un papel importante como variables significativas para adquirir y mantener conductas preventivas de embarazos no deseados a temprana edad y de infecciones de transmisión sexual (VIH/ SIDA), así como para promover la adopción de comportamientos sexuales saludables (Barrera, Sarmiento \& Vargas, 2004; Fernandez-Esquer, Atkinson, Diamond, Useche \& Mendiola, 2004; Fuensanta \& De la Rubia, 2001; Vargas-Trujillo, Barrera, Burgos \& Daza, 2006).

Las investigaciones y aproximaciones de las diferentes teorías sobre las actitudes, creencias y el enfoque social, han contribuido en forma impactante sobre la literatura en la realización de numerosos estudios y avances con respecto a la sexualidad reproductiva de adolescentes (BelloVillanueva et al., 2014; Collazos, Echeverri, Molina, Canaval \& Valencia, 2005; Crosby, Yarber \& Meyerson, 2000; Gilmore et al., 2002; Kelly, 2000; Soravisutr, 2002; Vargas-Trujillo \& Barrera, 2003; Whitaker et al., 2000).

En particular, la teoría del comportamiento planeado de Ajzen $(1991,2002)$ y Fishbein y Ajzen (1975) ha sido validada y ha resultado consistente en diferentes estudios (Chan \& Fishbein, 1993; Norris \& Ford, 1995; Vargas-Trujillo \& Barrera, 2003). Dicha teoría dimensiona las creencias, actitudes y normas subjetivas como elementos explicativos y predictivos del comportamiento sexual, que actúan como variables motivadoras fundamentales en la adquisición de una conducta (Oblitas, 2004).

No obstante, según apuntan diversas investigaciones nacionales e internacionales, los resultados de las escalas que evalúan actitudes y creencias en adolescentes varones reportan hallazgos inconsis- 
tentes que difieren en la relación de estas variables con la conducta sexual de riesgo, al igual que con los factores explicativos y predictivos en los comportamientos sexuales en esta población (Flórez, 2007; Gilmore et al., 2002; Guchin \& Meré, 2004; Orgen, 2003; Perdersen, Samuelsen \& Wichstrom, 2003).

Existen pocos instrumentos validados con enfoque específico para cada género; así mismo, en algunos estudios se encuentran fallas en el diseño de instrumentos de evaluación que carecen de análisis estadísticos rigurosos y basan los resultados solamente en encuestas no válidas científicamente (Franklin, Grant \& Corcoran, 1997).

En este mismo sentido, se reportan pocas investigaciones con adolescentes varones que evalúen actitudes, creencias y comportamiento en el campo de la salud sexual y reproductiva. Esta brecha es significativamente notoria frente a la numerosa literatura existente sobre adolescentes mujeres en América Latina (Infesta Domínguez, 2005; Lundgren, 2000; Madiedo, 2001; Páramo, 2011; Valdivia $\&$ Vizcarra, en prensa), se requieren de estudios que profundicen en la salud reproductiva de varones (Quiroz \& Henríquez, 2009) a pesar de la existencia de numerosas encuestas en los centros de control de enfermedades, no están diseñadas para abarcar la variedad de temáticas de salud sexual y reproductiva pertinente a los adolescentes varones, de conocimiento importante para el diseño de intervenciones en este ámbito. Se requieren de elementos que guíen el desarrollo de políticas en salud y programas efectivos para el trabajo con el género masculino (Infesta Domínguez, 2005; Pantelides \& López, 2005; Sapién \& Córdoba, 2011).

Tomando como base los planteamientos anteriores en la sexualidad reproductiva en los adolescentes varones se plantea el objetivo de los dos estudios en esta revisión: la construcción de una escala como prueba piloto y prueba definitiva para evaluar actitudes y creencias normativas como dimensiones pertinentes en el comportamiento sexual y reproductivo en varones.

Para la consecución del objetivo en mención tanto en la prueba piloto como en la definitiva e instrumento final, esta investigación se fundamentó en estudios realizados previamente por el grupo de investigadores del Centro de Investigaciones en desarrollo humano (CIDHUM) y de Comunicación y Cultura de la Universidad del Norte (Bello, Altamar, Mosquera \& Palacio, 2010; Bello, Gómez, Mosquera \& Palacio, 2010; Bello, Mosquera, Palacio \& Peralta, 2010; Mosquera et al., 2004) y por los hallazgos obtenidos en investigación a nivel nacional (Vega, 2009) con alianza de cooperación con la Consejería Presidencial de Programas Especiales (CPPE), el Programa de Promoción de Derechos y Redes Constructoras de Paz (PPDRCP), la Estrategia Red de Gestores Sociales (RGS); la Fundación Imaginario, Citurna Producciones, La Iniciativa de Comunicación y la Universidad del Norte.

\section{Estudio 1: fase piloto}

El objetivo del estudio piloto fue analizar y valorar aspectos de administración de la prueba comprensión de los ítems, tiempo de duración, etc.), y así conseguir una apreciación inicial de la confiabilidad, a través del análisis de consistencia interna Alfa de Cronbach.

\section{Método}

\section{Participantes}

Los participantes en este estudio piloto fueron 50 hombres adolescentes de la región Caribe, entre 11 y 19 años de edad, con una media de 14.20 y una DE de 1.877. Pertenecientes a un nivel socioeconómico medio-bajo de una institución pública.

Los criterios que se consideraron para seleccionar la muestra fue: ciudad representativa de la región Caribe; promedio de ICFES MEDIO en el 2009, para incrementar un equilibrio académico; instituciones mixtas públicas de estrato bajo con estudiantes entre 11 y 19 años pertenecientes a grados de escolaridad entre $6^{\circ}$ a $11^{\circ}$.

La muestra piloto fue de tipo no probabilística y de sujetos voluntarios (Hernández-Sampieri, Fernández-Collado \& Baptista-Lucio, 2010), quienes previamente firmaron un consentimiento informado junto a sus padres y/o acudientes. 
Ana Mercedes Bello-Villanueva, Jorge Palacio, Pablo Vera-Villarroel, Oscar Oviedo-Trespalacios, Melissa Rodríguez-Diaz, Karen Celis-Atenas, Paula Pavez.

\section{Variables}

La estructura y descripción de las escalas del instrumento, en la fase del pilotaje y en la fase de instrumento definitivo fueron las siguientes:

Creencias; se distinguen según la teoría de comportamiento planeado tres tipos de creencias: conductuales (que influyen y dirigen el comportamiento), normativas (la opinión de referentes importantes sobre la realización de determinada conducta influye en la norma subjetiva del sujeto) y de control (relacionadas con la dificultad o facilidad percibida para la ejecución de la conducta (Madden, Scholder, Pamela \& Ajzen, 1992). Las creencias normativas influyen sobre la norma subjetiva percibida provocando cambios en dicho comportamiento. Están caracterizadas como factor social que se refiere a la presión social percibida para realizar o no el comportamiento (Ajzen, 2001), reflejan la influencia que el entorno inmediato al sujeto ejerce sobre la conducta (Fishbein \& Ajzen, 1975).

En el presente estudio se enfatizo en las creencias normativas. Las creencias estarán dadas en relación a las normas subjetivas en función de las creencias que el joven tienen acerca de cuál es la opinión de “otras personas o grupos de referencia importantes para él (padres, profesores y amigos), con respecto a si debe realizar o no el comportamiento sexual y reproductivo (Ajzen, 2001). Teniendo en cuenta cuatro dimensiones (aprobación y apoyo de padres, profesores y amigos, disposición a complacer en métodos de protección, abstinencia y retardo en el inicio de relaciones sexuales e importancia de dichas figuras significativas en su sexualidad).

Las actitudes, se definen como una predisposición aprendida para responder de forma consistente, favorable o desfavorablemente con respecto a un objeto dado; la creencia sobre los objetos actitudinales se forma mediante la asociación de dicho objeto con determinados atributos (Ajzen, 2001). Abarca tres elementos fundamentales: actitud como consistencia, como predisposición a la respuesta y como disposición aprendida (Fishbein $\&$ Ajzen, 1975).

Este estudio entenderá las actitudes de acuerdo a la definición de Fishbein y Ajzen (1975), como la evaluación que el individuo hace favorable o desfavorablemente hacia la actividad sexual y comportamiento de protección reproductiva mediante adjetivos bipolares (necesario, saludable, maduro, seguro, responsable).

\section{Procedimiento}

La construcción de la prueba piloto de evaluación de actitudes y normas subjetivas sobre la sexualidad reproductiva en adolescentes varones adquirió el formato de escala Likert con puntuaciones de 1 a 5 y el procedimiento fue el siguiente:

1. Identificación de las dimensiones: se definieron las dimensiones de evaluación que debe contemplar el instrumento, para lo que se revisó literatura pertinente y actualizada, además se le consultó a jueces expertos a nivel nacional en el tema y a sujetos que cumplían con las características de la muestra.

2. Construcción de ítems: en la primera fase el instrumento llamado (ANCIP), diseñado por el grupo de investigadores, con base en los constructos teóricos especificados por la teoría de comportamiento planeado (Ajzen \& Fishbein, 2001); tuvo como objetivo identificar actitudes y creencias en adolescentes tanto varones como mujeres. Se realizó previamente un pilotaje de las creencias personales, de control y grupos de referencia, mediante entrevistas cognitivas y encuesta estructurada para medir con los mismos parámetros del contexto al grupo seleccionado para la muestra.

Con respecto a la validez del contenido se realizó acuerdo ínter jueces, definido el nivel de acuerdo ínter jueces se eligió únicamente a aquellos ítems que tuvieran validez de contenido muestral, quedando el instrumento confeccionado con 110 ítems distribuidos: 70 ítems de creencias y 40 de actitudes.

3. Aplicación Prueba piloto: en la segunda fase se procedió a la aplicación de esta prueba con algunas modificaciones, se realizó en forma independiente los cuestionarios para la versión tanto masculina como femenina, después de seleccionar los ítems con validez de contenido muestral, teniendo en cuenta el acuerdo interjueces a nivel nacional e internacional. La forma masculina, finalmente 
estuvo constituida por 150 ítems, 72 ítems de actitudes y 78 de normas subjetivas en una muestra de 50 adolescentes varones.

\section{Análisis de Datos}

El análisis de los datos se realizó a través del Paquete Estadístico para Ciencias Sociales (SPSS), versión 19 para Windows. La confiabilidad se estimó a partir del Alfa de Cronbach, mientras que la validez a partir del análisis factorial exploratorio.

\section{Resultados}

\section{Fiabilidad}

La apreciación inicial de la confiabilidad para los ítems de actitud relacionados con la prevención del embarazo e infecciones de transmisión sexual, se obtuvo un Alfa de Cronbach que oscila entre 0.94 y 0.97 .

En cuanto a los grupos de ítems relacionados con la escala normas subjetivas, el análisis de consistencia interna reporta en cada una de sus dimensiones un Alfa de Cronbach de 0.94 mientras que en la dimensión de aprobación y de apoyo, en disposición a complacer a otros y en la dimensión de importancia fue de 0.95 .

Mediante el coeficiente Alfa de Cronbach se estimó la confiabilidad para el total de la escala de actitud resultando un $\alpha=0.97$ y un $\alpha=0.98$ para el total de la escala de normas subjetivas.

Se eliminaron aquellos ítems que no aportaban a la confiabilidad de la escala, descartando16 reactivos para las creencias normativas y 56 para actitudes. Una vez realizado este procedimiento, se estimó nuevamente la confiabilidad, arrojando el siguiente Alfa de Cronbach para la escala total de actitud $\alpha=0.92$ y para la escala total de normas subjetivas los coeficientes alfa reportan $\alpha=0.97$.

\section{Análisis factorial}

En la escala de actitud el análisis factorial exploratorio por componentes principales evidencia que se extraen en total 15 factores que son reagrupados de la siguiente manera: para el grupo de ítems relacionados con la actitud hacia la protección se extraen 5 que se explican en un $78.93 \%$ en el segundo grupo de ítems referidos a la actitud hacia la abstinencia se extraen 4 factores explicados en un $78.23 \%$ y en el tercer grupo correspondiente a actitud hacia el retardo en el inicio de la relación sexual se extraen 6 factores explicados en $78.92 \%$ respectivamente.

En el análisis de los componentes principales en las creencias normativas se extraen 4 factores explicados en un $80.08 \%$ en dimensión aprobación; para los ítems de apoyo se extraen 5 factores que se explican en un $80.93 \%$. En la dimensión de disposición se extraen 3 componentes explicados en un $78.12 \%$ y para la dimensión de importancia se observan 4 componentes extraídos que se explican en un $81.41 \%$.

\section{Estudio 2: Prueba Definitiva}

El propósito de este segundo estudio fue obtener indicadores definitivos de la confiabilidad y validez de las escalas de actitud y de creencias normativas en la población de adolescentes escolarizados en las cinco ciudades representativas de la región Caribe.

\section{Método}

\section{Participantes}

Para este estudio se seleccionaron 915 varones de la región Caribe, entre 11 y 19 años de edad, con una media de 14.16 y una desviación estándar de 2.11. Pertenecientes a un nivel socioeconómico medio-bajo, de 15 colegios de la región Caribe, en Colombia. La muestra definitiva fue de tipo no probabilística y de sujetos voluntarios (HernándezSampieri et al., 2010). Los criterios de inclusión fueron similares a los del estudio 1 .

\section{Procedimiento}

Una vez se analizo la prueba piloto, se sometió nuevamente a validación por jueces expertos previamente a la aplicación final. Se consulto a 9 profesionales con experticia en esta temática y en 
Ana Mercedes Bello-Villanueva, Jorge Palacio, Pablo Vera-Villarroel, Oscar Oviedo-Trespalacios, Melissa Rodríguez-Diaz, Karen Celis-Atenas, Paula Pavez.

el diseño de pruebas psicométricas, pero solo se obtuvo respuesta de 5 jueces expertos provenientes de universidades internacionales y nacionales: California State University, Fresno (Universidad de Fresno California), Universidad Nacional Autónoma de México UNAM; Instituto Mexicano de Investigación de Familia y Población (IMIFAP); Universidad de los Andes, en Bogotá y Universidad Pontifica Bolivariana, en Medellín. Posteriormente se hicieron ajustes antes de la aplicación final.

Aplicación definitiva del instrumento: finalmente la prueba se administró a los sujetos seleccionados. El cuestionario en su versión final quedó conformado por un total de 78 ítems correspondiente a 16 ítems en la escala de actitud hacia la protección, abstinencia y retardo en el inicio de las relaciones sexuales y 62 ítems que evalúan creencias en cuanto a normas subjetivas debido a que se consideró que los ítems ofrecen información útil. La prueba fue administrada por 30 encuestadores previamente entrenados, en un tiempo promedio de 45 minutos por persona.

\section{TABLA 1}

Variables sociodemográficas de la muestra*

\begin{tabular}{cc}
\hline Características & No. (\%) \\
\hline Edad (años) & \\
\hline Media (DE) & $14.16(2.11)$ \\
$10-13$ & 378 \\
$14-16$ & 408 \\
$17-19$ & 128 \\
\hline Grado Escolaridad & \\
\hline Media (DE) & $8.28(1.8)$ \\
$6-7$ & 333 \\
$8-9$ & 300 \\
$10-11$ & 278 \\
\hline Ciudad & \\
\hline Barranquilla & 281 \\
Santa Marta & 293 \\
Cartagena & 128 \\
Montería & 127 \\
Riohacha & 86 \\
\hline
\end{tabular}

(*) $N=915$

Fuente: elaboración propia.

\section{Resultados}

Se tuvieron en cuenta variables sociodemográficas en la muestra que se relacionan con la edad, escolaridad y ciudad de origen y se observan en la Tabla 1.

\section{Análisis fiabilidad}

\section{Escala de Actitud}

La media obtenida en la escala de actitud hacia la protección fue de $4.1(D E=1.07)$, el alfa de Cronbach obtenido para esta escala fue de 0.93 , con respecto a la actitud hacia la abstinencia y retardo la media fue de $3.6(D E=1.26)$, mientras que el alfa de 0.88; la evaluación de la homogeneidad fue elevada en todos los casos obteniéndose correlación ítem total superiores a 0.6. Véase Tabla 2 donde se detallan media, desviación estándar y correlación ítem total para cada uno de los ítems de la escala de actitud.

\section{Escala Normas Subjetivas}

Para la escala de normas subjetivas se obtuvo una media de $3.79(D E=1.08)$, la estimación de la confiabilidad por medio de alfa de Cronbach fue de 0.98 . El promedio de la correlación ítem total fue de 0.74 con un valor mínimo de 0.66 y máximo 0.82 y $D E=0.04$, en la mayoría de los casos superaron el valor sugerido de 0.6 (Hair, Black, Babin \& Anderson, 2010).

La estimación de la consistencia interna para cada una de las dimensiones fue de 0.96 dimensión de aprobación y apoyo, de 0.94 para la dimensión Dispuesto y finalmente para la dimensión de Importancia fue de 0.94; la homogeneidad de la prueba fue adecuada con correlaciones superiores a $\mathbf{0 . 6 6}$, en la Tabla 3 se detallan media, desviación estándar y correlación ítem-total para cada una de las dimensiones del cuestionario (Aprobación y Apoyo, Dispuesto, Importancia). 
TABLA 2

Confiabilidad ítem-escala de actitud del "Cuestionario salud sexual y reproductiva- Forma Masculina"*

\begin{tabular}{|c|c|c|c|c|}
\hline \multirow[b]{2}{*}{ Escala } & \multicolumn{3}{|c|}{ Consistencia Interna } & \multirow{2}{*}{$\begin{array}{l}\text { Confiabilidad (alfa } \\
\text { de Cronbach) }\end{array}$} \\
\hline & $\begin{array}{c}\text { Promedio Correlación } \\
\text { ítem-ítem }\end{array}$ & $\begin{array}{c}\text { Mínimo Correlación } \\
\text { ítem-ítem }\end{array}$ & $\begin{array}{c}\text { Máximo Correlación } \\
\text { ítem-ítem }\end{array}$ & \\
\hline \multicolumn{5}{|l|}{ Actitud } \\
\hline Actitud Protección & 0.62 & 0.55 & 0.81 & 0.94 \\
\hline $\begin{array}{l}\text { Actitud Abstinencia/ } \\
\text { retardo }\end{array}$ & 0.51 & 0.42 & 0.69 & 0.88 \\
\hline
\end{tabular}

$* \mathrm{~N}=915$

Fuente: elaboración propia.

TABLA 3

Confiabilidad item-escala de Normas Subjetivas del "Cuestionario salud sexual y reproductiva- Forma Masculina" *

\begin{tabular}{|c|c|c|c|c|}
\hline \multirow[b]{2}{*}{ Escala } & \multicolumn{3}{|c|}{ Consistencia Interna } & \multirow[b]{2}{*}{$\begin{array}{c}\text { Confiabilidad } \\
\text { (alfa de } \\
\text { Cronbach) }\end{array}$} \\
\hline & $\begin{array}{l}\text { Promedio } \\
\text { Correlación } \\
\text { ítem-ítem }\end{array}$ & $\begin{array}{c}\text { Mínimo } \\
\text { Correlación } \\
\text { ítem-ítem }\end{array}$ & $\begin{array}{c}\text { Máximo } \\
\text { Correlación } \\
\text { ítem-ítem }\end{array}$ & \\
\hline Aprobación y apoyo de personas adultas protección (AE, AO) & 0.64 & 0.54 & 0.82 & 0.95 \\
\hline Aprobación y apoyo de padres en abstinencia/retardo & 0.57 & 0.48 & 0.7 & 0.89 \\
\hline Aprobación y apoyo de profesores en abstinencia/retardo & 0.61 & 0.52 & 0.68 & 0.89 \\
\hline Aprobación y apoyo de amigos abstinencia/retardo & 0.58 & 0.5 & 0.67 & 0.89 \\
\hline Aprobación y apoyo de amigos protección $(\mathrm{AE}, \mathrm{AO})$ & 0.68 & 0.58 & 0.73 & 0.93 \\
\hline $\begin{array}{l}\text { Dispuesto a complacer padres, profesores y amigos en } \\
\text { abstinencia/retardo }\end{array}$ & 0.61 & 0.49 & 0.74 & 0.92 \\
\hline $\begin{array}{l}\text { Dispuesto a complacer padres, profesores y amigos en } \\
\text { protección (AE, AO) }\end{array}$ & 0.68 & 0.63 & 0.71 & 0.91 \\
\hline $\begin{array}{l}\text { Importancia de padres, profesores y amigos en abstinencia/ } \\
\text { retardo }\end{array}$ & 0.55 & 0.43 & 0.71 & 0.92 \\
\hline Importancia de padres, profesores y amigos en protección & 0.61 & 0.49 & 0.79 & 0.9 \\
\hline
\end{tabular}

$* N=915$

Fuente: elaboración propia.

\section{Análisis factorial}

\section{Escala de Actitud}

La evaluación de la validez de constructo se realizó por medio del análisis factorial exploratorio y análisis de componentes principales con rotación VARIMAX. La adecuación de los datos a la matriz factorial fue satisfactoria en cada una de las dimensiones, se encontró en la escala de actitud un $\mathrm{KMO}=0.92$ y la prueba de esfericidad de Bartlett fue altamente significativa $\left(\chi^{2}\right.$
$=8712.24 ; g l=120 ; p<0.01)$. Se extrajeron 2 factores que se explica en un $62.62 \%$ de la varianza (Hair et al., 2010) consideran que un valor total de varianza explicada por encima del $60 \%$ es satisfactorio. La estructura factorial está compuesta por dos grupos de ítems agrupados en un primer factor 9 ítems pertenecientes a la dimensión actitud hacia la protección y en el segundo factor un grupo de 7 ítems correspondientes a la actitud hacia la abstinencia y retardo en el inicio de la relación, en la Tabla 4 véase el detalle de solución factorial. 
Ana Mercedes Bello-Villanueva, Jorge Palacio, Pablo Vera-Villarroel, Oscar Oviedo-Trespalacios, Melissa Rodríguez-Diaz, Karen Celis-Atenas, Paula Pavez.

TABLA 4

Medias, desviaciones, correlaciones ítem-total y análisis factorial: componentes principales con rotación VARIMAX Escala de Actitud

\begin{tabular}{lccccc}
\hline \multicolumn{1}{c}{ Ítem } & Media & DE & \multicolumn{2}{c}{ Correlación } & Componentes \\
& Ittem-Total & 1 & 2 \\
\hline Usar condón evitar embarazo es RESPONSABLE & 4.05 & 1.2 & 0.74 & 0.8 \\
Usar condón evitar ITS es RESPONSABLE & 4.15 & 1.07 & 0.76 & 0.79 \\
Usar condón evitar ITS es NECESARIO & 4.18 & 1.02 & 0.73 & 0.78 \\
Usar condón evitar embarazo es SALUDABLE & 3.99 & 1.08 & 0.76 & 0.78 \\
Usar condón evitar ITS es SALUDABLE & 4.15 & 1.04 & 0.77 & 0.78 \\
Usar condón evitar embarazo es MADURO & 4.04 & 1.05 & 0.76 & 0.77 \\
Usar condón evitar ITS es MADURO & 4.13 & 1.04 & 0.77 & 0.76 \\
Usar condón evitar embarazo es SEGURO & 4.03 & 1.03 & 0.78 & 0.75 \\
Usar condón evitar ITS es SEGURO & 4.14 & 1.05 & 0.76 & 0.75 \\
Evitar relaciones adolescencia ITS SALUDABLE & 3.53 & 1.31 & 0.7 & & 0.76 \\
Posponer relaciones 18 años ITS SALUDABLE & 3.60 & 1.23 & 0.6 & & 0.76 \\
Posponer relaciones 18 años ITS SEGURO & 3.67 & 1.26 & 0.6 & & 0.75 \\
Posponer relaciones 18 años embarazo MADURO & 3.59 & 1.2 & 0.62 & & 0.75 \\
Evitar relaciones adolescencia embarazo MADURO & 3.55 & 1.28 & 0.72 & & 0.69 \\
Evitar relaciones adolescencia ITS MADURO & 3.69 & 1.2 & 0.71 & & 0.68 \\
Evitar relaciones adolescencia embarazo SEGURO & 3.57 & 1.33 & 0.71 & & 0.66 \\
\hline Porcntaje val & & & &
\end{tabular}

Porcentaje de variabilidad explicado $=62.62$

Fuente: elaboración propia.

\section{Escala Normas Subjetivas}

La evaluación de la dimensionalidad de la escala se realizó por medio del análisis factorial exploratorio por componentes principales y rotación VARIMAX. El conjunto de ítem se adecuada a la matriz factorial, encontrándose la prueba de adecuación muestral de Kaiser-Meyer-Olkin en un $\mathrm{KMO}=$ 0.97 y altamente significativa el test de esfericidad de Bartlett $\left(\chi^{2}=40933.311 ; g l=1891 ; p<0.01\right)$. Se extrajeron 9 factores que se explican $68.59 \%$ de la varianza. En las Tablas 5 a y 5 b se detalla la solución factorial.

El análisis factorial contribuyo en la distribución de los ítems en la prueba final considerando todas las cargas factoriales por arriba de 0.4 y quedo establecida en una escala de 78 ítems tipo Likert, frente a la que los sujetos deben responder entre 5 número de alternativas, en donde solo debe marcarse una opción de respuesta, que van desde la afirmación nada saludable hasta muy saludable, nada seguro hasta muy seguro, nada importante hasta muy importante y desde muy en desacuerdo hasta muy de acuerdo. Las afirmaciones pueden indicar dirección favorable o positiva y/o desfavorable o negativa.

\section{Discusión}

La escala de creencias y actitudes en comportamiento sexual y reproductivo se construye a partir de la inexistencia de instrumentos validados con un enfoque específico para cada género, y de los hallazgos de algunas investigaciones que indican la existencia de diversidad y variaciones que se reflejan en el fenómeno estudiado, en cuanto al análisis de constructos que detecten un análisis diferencial de géneros y faciliten esta diversidad en los procesos educativos institucionales y en programas de salud sexual (Álvarez-Gayou, Honold, Millán \& Ortega, 2004; Hidalgo San Martin, Caballero-Hoyos, Celis De la Rosa \& Rasmussen-Cruz, 2003; Infesta Domínguez, 1998; Zárate \& Calderón, 2010). 


\section{TABLA 5A}

Medias, desviaciones, correlaciones item-total y análisis factorial: componentes principales con rotación VARIMAX para la escala de Normas Subjetivas

\begin{tabular}{|c|c|c|c|c|c|c|c|c|c|c|c|}
\hline \multirow{2}{*}{ Ítems } & \multirow{2}{*}{ Media } & \multirow{2}{*}{$\mathrm{DE}$} & \multirow{2}{*}{$\begin{array}{l}\text { Correlación } \\
\text { Ítem-Total }\end{array}$} & \multicolumn{8}{|c|}{ Componentes } \\
\hline & & & & 1 & 2 & 3 & 4 & 5 & 67 & 78 & 9 \\
\hline \multicolumn{12}{|l|}{ Aprobación y apoyo de Adultos protección (AE, AO) } \\
\hline Padres aprobarían usar condón embarazo. & 3.80 & 1.3 & 0.81 & 0.79 & & & & & & & \\
\hline Padres aprobarían usar condón evitar ITS. & 3.98 & 1.13 & 0.81 & 0.79 & & & & & & & \\
\hline Padres apoyarían usar condón embarazo. & 3.91 & 1.13 & 0.8 & 0.77 & & & & & & & \\
\hline Padres apoyarían usar condón evitar ITS. & 4.03 & 1.04 & 0.8 & 0.76 & & & & & & & \\
\hline Padres acuerdo que jóvenes usen condón embarazo. & 3.95 & 1.12 & 0.75 & 0.75 & & & & & & & \\
\hline Padres acuerdo que jóvenes condón evitar ITS. & 4.03 & 1.11 & 0.76 & 0.75 & & & & & & & \\
\hline Profesores aprobarían usar condón embarazo. & 3.77 & 1.13 & 0.79 & 0.74 & & & & & & & \\
\hline Profesores aprobarían usar condón evitar ITS. & 3.92 & 1.05 & 0.81 & 0.74 & & & & & & & \\
\hline Profesores apoyarían usar condón embarazo. & 3.86 & 1.07 & 0.77 & 0.68 & & & & & & & \\
\hline Profesores apoyarían usar condón evitar ITS. & 3.99 & 1.01 & 0.73 & 0.68 & & & & & & & \\
\hline Profesores acuerdo que jóvenes usen condón embarazo. & 3.66 & 1.33 & 0.78 & 0.66 & & & & & & & \\
\hline Profesores acuerdo que jóvenes condón evitar ITS. & 3.88 & 1.12 & 0.75 & 0.63 & & & & & & & \\
\hline \multicolumn{12}{|l|}{ Aprobación y apoyo de amigos protección (AE, $\mathrm{AO})$} \\
\hline Amigos aprobarían usar condón embarazo. & 3.62 & 1.14 & 0.77 & & 0.65 & & & & & & \\
\hline Amigos aprobarían usar condón evitar ITS. & 3.71 & 1.16 & 0.82 & & 0.61 & & & & & & \\
\hline Amigos apoyarían usar condón embarazo. & 3.68 & 1.11 & 0.81 & & 0.6 & & & & & & \\
\hline Amigos apoyarían usar condón evitar ITS. & 3.79 & 1.09 & 0.79 & & 0.59 & & & & & & \\
\hline Amigos de acuerdo que jóvenes usen condón embarazo. & 3.68 & 1.14 & 0.79 & & 0.56 & & & & & & \\
\hline Amigos de acuerdo que jóvenes usen condón evitar ITS. & 3.78 & 1.12 & 0.74 & & 0.54 & & & & & & \\
\hline \multicolumn{12}{|l|}{ Aprobación y apoyo de padres abstinencia/retardo } \\
\hline Padres aprobarían evitar relaciones adolesc. ITS. & 3.54 & 1.38 & 0.72 & & & 0.7 & & & & & \\
\hline Padres aprobarían posponer relaciones 18 años embarazo. & 3.75 & 1.22 & 0.76 & & & 0.68 & & & & & \\
\hline Padres aprobarían posponer relaciones 18 años ITS. & 3.57 & 1.14 & 0.67 & & & 0.67 & & & & & \\
\hline Padres apoyarían evitar relaciones adolesc. embarazo. & 3.69 & 1.16 & 0.7 & & & 0.66 & & & & & \\
\hline Padres aprobarían evitar relaciones adolesc. embarazo. & 3.77 & 1.19 & 0.67 & & & 0.64 & & & & & \\
\hline Padres apoyarían evitar relaciones adolesc. ITS. & 3.87 & 1.19 & 0.7 & & & 0.61 & & & & & \\
\hline \multicolumn{12}{|l|}{ Aprobación y apoyo de profesores abstinencia/retardo } \\
\hline Profesores aprobarían evitar relaciones adolesc. embarazo. & 3.48 & 1.21 & 0.76 & & & & 0.71 & & & & \\
\hline Profesores aprobarían posponer relaciones 18 años embarazo. & 3.52 & 1.17 & 0.66 & & & & 0.69 & & & & \\
\hline Profesores aprobarían posponer relaciones 18 años ITS. & 3.53 & 1.21 & 0.75 & & & & 0.68 & & & & \\
\hline Profesores apoyarían evitar relaciones adolesc. embarazo. & 3.57 & 1.16 & 0.73 & & & & 0.64 & & & & \\
\hline Profesores apoyarían evitar relaciones adolesc. ITS. & 3.61 & 1.22 & 0.74 & & & & 0.63 & & & & \\
\hline \multicolumn{12}{|l|}{ Aprobación y apoyo de amigos abstinencia/retardo } \\
\hline Amigos aprobarían evitar relaciones adolesc. ITS. & 3.22 & 1.16 & 0.67 & & & & & 0.76 & & & \\
\hline Amigos aprobarían posponer relaciones 18 años embarazo. & 3.04 & 1.18 & 0.71 & & & & & 0.74 & & & \\
\hline Amigos apoyarían evitar relaciones adolesc. embarazo. & 3.19 & 1.22 & 0.72 & & & & & 0.74 & & & \\
\hline Amigos apoyarían evitar relaciones adolesc. ITS. & 3.32 & 1.15 & 0.75 & & & & & 0.74 & & & \\
\hline Amigos apoyarían posponer relaciones 18 años embarazo. & 3.15 & 1.20 & 0.75 & & & & & 0.71 & & & \\
\hline Amigos apoyarían posponer relaciones 18 años ITS. & 3.35 & 1.15 & 0.7 & & & & & 0.67 & & & \\
\hline
\end{tabular}

Fuente: elaboración propia.

Ahora bien, las administraciones prácticas y funcionales de la escala señalan la utilidad como herramienta de evaluación y diagnostico de las actitudes y creencias que facilitan y garantizan un óptimo comportamiento sexual y reproductivo en los jóvenes adolescentes. En este mismo sentido, 
Ana Mercedes Bello-Villanueva, Jorge Palacio, Pablo Vera-Villarroel, Oscar Oviedo-Trespalacios, Melissa Rodríguez-Diaz, Karen Celis-Atenas, Paula Pavez.

\section{TABLA 5B}

Medias, desviaciones, correlaciones ítem-total y análisis factorial: componentes principales con rotación VARIMAX para la Escala de Normas Subjetivas

\begin{tabular}{|c|c|c|c|c|c|c|c|c|c|}
\hline \multirow{2}{*}{ Ítems } & \multirow{2}{*}{ Media } & \multirow{2}{*}{$\mathrm{DE}$} & \multirow{2}{*}{$\begin{array}{c}\text { Correlación } \\
\text { Ítem-Total }\end{array}$} & \multicolumn{6}{|c|}{ Componentes } \\
\hline & & & & 123 & 45 & 6 & 7 & 8 & 9 \\
\hline \multicolumn{10}{|l|}{$\begin{array}{l}\text { Dispuesto a complacer padres, profesores y amigos en } \\
\text { protección }(\mathrm{AE}, \mathrm{AO})\end{array}$} \\
\hline Dispuesto complacer padres usar condón evitar ITS & 3.91 & 1.11 & 0.78 & & & 0.43 & & & \\
\hline Dispuesto complacer profesores usar condón embarazo & 3.74 & 1.15 & 0.8 & & & 0.52 & & & \\
\hline Dispuesto complacer profesores usar condón evitar ITS & 3.9 & 1.08 & 0.78 & & & 0.5 & & & \\
\hline Dispuesto complacer amigos usar condón embarazo & 3.72 & 1.18 & 0.76 & & & 0.65 & & & \\
\hline Dispuesto complacer amigos usar condón evitar ITS & 3.88 & 1.11 & 0.76 & & & 0.59 & & & \\
\hline \multicolumn{10}{|l|}{ Dispuesto a complacer padres, profesores y amigos en abstinencia/retardo } \\
\hline Dispuesto complacer padres evitar relaciones embarazo & 3.26 & 1.38 & 0.79 & & & 0.71 & & & \\
\hline Dispuesto complacer padres evitar relaciones ITS & 3.48 & 1.28 & 0.78 & & & 0.7 & & & \\
\hline Dispuesto complacer padres posponer relaciones 18 años embarazo & 3.36 & 1.25 & 0.72 & & & 0.68 & & & \\
\hline Dispuesto complacer profesores evitar relaciones embarazo & 3.26 & 1.29 & 0.79 & & & 0.68 & & & \\
\hline Dispuesto complacer profesores evitar relaciones ITS & 3.46 & 1.2 & 0.76 & & & 0.55 & & & \\
\hline Dispuesto complacer profesores posponer relaciones 18 años embarazo & 3.33 & 1.22 & 0.72 & & & 0.55 & & & \\
\hline Dispuesto complacer amigos evitar relaciones ITS & 3.3 & 1.21 & 0.66 & & & 0.54 & & & \\
\hline \multicolumn{10}{|l|}{ Importancia de padres, profesores y amigos en abstinencia/retardo } \\
\hline Importante padres piensen posponer relaciones 18 años embarazo & 3.64 & 1.17 & 0.7 & & & & & 0.65 & \\
\hline Importante padres piensen posponer relaciones 18 años ITS & 3.82 & 1.16 & 0.71 & & & & & 0.63 & \\
\hline Importante profesores piensen evitar relaciones embarazo & 3.55 & 1.19 & 0.72 & & & & & 0.55 & \\
\hline Importante profesores piensen evitar relaciones ITS & 3.65 & 1.17 & 0.73 & & & & & 0.55 & \\
\hline Importante profesores piensen posponer relaciones 18 años embarazo & 3.55 & 1.17 & 0.74 & & & & & 0.72 & \\
\hline Importante profesores piensen posponer relaciones 18 años ITS & 3.64 & 1.18 & 0.74 & & & & & 0.68 & \\
\hline Importante amigos piensen evitar relaciones ITS & 3.49 & 1.18 & 0.66 & & & & & 0.54 & \\
\hline Importante amigos piensen posponer relaciones 18 años embarazo & 3.39 & 1.19 & 0.68 & & & & & 0.74 & \\
\hline Importante amigos piensen posponer relaciones 18 años ITS & 3.53 & 1.21 & 0.7 & & & & & 0.63 & \\
\hline \multicolumn{10}{|l|}{ Importancia de padres, profesores y amigos en protección } \\
\hline Importante padres piensen condón embarazo & 3.82 & 1.26 & 0.73 & & & & & & 0.56 \\
\hline Importante padres piensen condón ITS & 4.04 & 1.08 & 0.77 & & & & & & 0.55 \\
\hline Importante profesores piensen condón embarazo & 3.86 & 1.03 & 0.79 & & & & & & 0.74 \\
\hline Importante profesores piensen condón ITS & 3.95 & 1.07 & 0.76 & & & & & & 0.55 \\
\hline Importante amigos piensen condón embarazo & 3.85 & 1.04 & 0.68 & & & & & & 0.62 \\
\hline Importante amigos piensen condón ITS & 3.94 & 1.06 & 0.7 & & & & & & 0.59 \\
\hline
\end{tabular}

Fuente: elaboración propia.

constituye una estrategia de formación orientada a facilitar cambios actitudinales y en el sistema de creencias con respecto a la sexualidad reproductiva de estos jóvenes escolares.

Los análisis realizados demuestran que las propiedades psicométricas del instrumento son adecuados e implican una evaluación valida y confiable, con consistencia interna alta y destacan su utilidad en la evaluación de actitudes y creencias (normas subjetivas) en los adolescentes varones en las dimensiones obtenidas, de acuerdo a los parámetros para las ciencias sociales (Hair et al., 2010). En consecuencia, esta investigación evidencia la existencia de la confiabilidad del cuestionario en dichos análisis.

La validación de la escala de actitud y creencias en salud sexual y reproductiva permitió utilizar criterios excelentes. Los resultados presentados 
anteriormente muestran los altos valores alcanzados en adolescentes varones y nos sugieren que los ítems se agrupan en una escala donde existe una alta correlación entre estos, con valores alcanzados en su gran mayoría en el alfa de Cronbach superiores a 0.88 que indican una confiabilidad muy aceptable.

El análisis de tipo exploratorio produjo la obtención de dos factores en las dimensiones de la escala de actitud que pese a su agrupación en tres factores en el pilotaje se explican mejor en la versión final del instrumento en la escala de actitud en dos componentes: actitud protección y actitud abstinencia y retardo. Estos últimos argumentan su agrupación debido a que comparten características similares en cuanto al método y la variación está dada por el tiempo de duración en la decisión de evitar tener relación sexual.

En la escala de creencias con respecto a la dimensión normas subjetivas, se sugiere una estructura adecuada al interior y altamente definida en este grupo de ítems en la escala. No obstante, se extrajeron 11 factores, de los cuales son interpretables 9 factores, a partir de la solución factorial, pertinentes y útiles para la validación del constructo fundamentados y correlacionados con el marco teórico cognitivo social.

El resultado del análisis factorial confirma la propuesta de la evaluación de jueces expertos en la selección de los ítems y temáticas que caracterizan cada una de las dimensiones de la escala. En general, con base en las evidencias de los resultados factoriales obtenidos que implican correlaciones altas entre los ítems y constructos, se permite concluir que el proceso de validación en relación a la validez del constructo y de consistencia interna reportan resultados que garantizan la legitimidad de la escala en poblaciones de adolescentes similares con respecto a edad, genero y grado de escolaridad.

Se espera continuar con este proceso de validación en estudios comparativos utilizando otros cuestionarios psicométricos, para así ajustar y reducir el número de ítems, minimizar el tiempo de administración de la escala y facilitar su aplicación.

\section{Referencias}

Ajzen, I. (1991). The theory of planned behavior. Organizational Behavior and Human Decission Processes, 50(2), 179-211.

Ajzen, I. (2001). Nature and operation of attitudes. Annual Review of Psychology, 52(1), 27-58.

Ajzen, I. (2002). Theory of planned behavior: Frequently asked questions. Recuperado el 11 de agosto de 2003, de http//www.people.umass.edu/aizen/faq. html

Álvarez-Gayou, J. L., Honold, E. J., Millán, A. P. \& Ortega, S. (2004). Escala de actitudes ante la propia sexualidad: un estudio de validación. Archivos Hispanoamericanos de Sexología,10(2), 151-165.

Barrera, F., Sarmiento, E. \& Vargas, E. (2004). Relación de las actitudes personales y de la norma social con la actividad sexual de los adolescentes. Revista de Estudios Sociales, 17, 56-66.

Bearman, P. \& Brückner, H. (1999). Peer effects on adolescent sexual debut and pregnancy: An analysis of a national survey of adolescent girls. In Power in numbers: Peer effects on adolescent girls' sexual debut and pregnancy (pp. 7-26). Washington, DC: The National Campaing to Prevent Teen Pregnancy.

Bello, A., Altamar, N., Mosquera, M. \& Palacio, J. (2010). Actitud, norma subjetiva, control percibido e intención hacia las prácticas de protección sexual para la prevención de embarazos no planeados e infecciones de transmisión sexual ITS/VIH/SIDA en adolescentes escolarizados de la ciudad de Barranquilla. Tesis de Magíster en Psicología no publicada, Universidad del Norte, Barranquilla, Colombia.

Bello, A., Gómez, J., Mosquera, M. \& Palacio, J. (2010). Actitud, norma subjetiva, control percibido e intención hacia las prácticas de protección sexual para la prevención de embarazos no planeados ITS y VIH/ SIDA en adolescentes escolarizados de Ciénaga. Tesis de Magíster en Psicología no publicada, Fundación Universidad del Norte, Barranquilla, Colombia.

Bello, A., Mosquera, M., Palacio, J. \& Peralta, S. (2010). Actitud, norma subjetiva, control percibido e intención hacia las prácticas de protección sexual para la prevención de embarazos no planeados ITS y VIH/ SIDA en adolescentes escolarizados del municipio de Sincelejo. Tesis de Magíster en Psicología no 
Ana Mercedes Bello-Villanueva, Jorge Palacio, Pablo Vera-Villarroel, Oscar Oviedo-Trespalacios, Melissa Rodríguez-Diaz, Karen Celis-Atenas, Paula Pavez.

publicada, Fundación Universidad del Norte, Barranquilla, Colombia.

Bello-Villanueva, A. M., Palacio, J., Vera-Villarroel, P., Oviedo-Trespalacios, O., Rodríguez-Diaz. M., Celis-Atenas, K., Pavez, P. (2014). Construcción y validación de una escala para evaluar salud sexual y reproductiva en adolescentes mujeres de la región caribe colombiana. Artículo en revisión.

Carvajal, S., Parcel, G., Banspach, S., Basen-Enqquist, K., Coyle, K., Kirby, D. \& Chan, W. (1999). Psychosocial predictors of delay of first sexual intercourse by adolescents. Health Psychology, 18(5), 443-452.

Collazos, M., Echeverri, N., Molina, A., Canaval, G. \& Valencia, C. (2005). Riesgo de VIH/Sida en la mujer: No es cuestión de estrato socioeconómico. Colombia Médica, 36(3), 50-57.

Crosby, R. A., Yarber, W. L. \& Meyerson, B. (2000). Prevention strategies other tan male condoms employed by low-income women to prevent HIV infection. Public Healt Nursing, 17(1), 53-60.

Chan, D. K. \& Fishbein, M. (1993). Determinants of college women's intentions to tell their partners to use condoms. Journal of Applied Social Psychology, 23(18), 1455-1470.

Di Clement, Crosby, R. \& Wingood, G. (2002). La prevención de VIH/SIDA en adolescentes. Deficiencias detectadas y enfoques nuevos. Perspectivas, 32(2), 1-23. Recuperado el 22 de julio de 2010, de http://www.ibe.unesco.org/publications/Prospects/ ProspectsPdf/122s/122s.pdf

Fernández-Esquer, M. E., Atkinson, J., Diamond, P., Useche, B. \& Mendiola, R. (2004). Condom use self-efficacy among U.S. - and foreign-born Latinos in Texas. The Journal of Sex Research, 41(4), 390-399.

Fishbein, M. \& Ajzen, I. (1975). Belief, attitude, intention, and behavior: An introduction to theory and research. Reading, MA: Addison-Wesley.

Flórez, A. (2007). Psicología social de la salud. Promoción y prevención. Bogotá: El Manual Moderno.

Franklin, C., Grant, D. \& Corcoran, J. (1997). Effectiveness of prevention programs for adolescent pregnancy: A meta-analysis. Journal of Marriage and the Family, 59(3), 551-567.
Fuensanta, L. R. \& De la Rubia, J. L. (2001). Validación de una escala de autoeficacia para la prevención del SIDA en adolescentes. Salud Pública de México, 43(5), 421-432.

Gilmore, M., Archibald, M., Morrison, D., Wilsdon, A., Wells, E., Hoppe, M. \& Murowchick, E. (2002). Teen sexual behavior: Applicability of the theory of reasoned action. Journal of Marriage and Family, 64(4), 885-897.

Guchin, M. \& Meré, J. (2004). Jóvenes, sexualidad y VIH/ SIDA en el Uruguay. Conocer para prevenir. Programa sexualidad y género. Montevideo, Uruguay: UNESCO, Iniciativa Latinoamericana.

Hair, J., Black, W., Babin, B. \& Anderson, R. (2010). Multivariate data analysis (6a. ed.). Upper Saddle River, NJ: Prentice-Hall.

Hernández-Sampieri, R., Fernández-Collado, C. \& Baptista-Lucio, M. (2010). Metodología de la investigación. Mexico: McGraw Hill.

Hidalgo San-Martin, A., Caballero-Hoyos, R., Celisde la Rosa, A. \& Rasmussen-Cruz, B. (2003). Validación de un cuestionario para el estudio del comportamiento sexual, social y corporal de adolecentes escolares. Salud Pública de México, 45(Supl. 1), S58-S72.

Infesta Domínguez, G. (1998, mayo). The implications of the researcher's gender in the construction of data for studies on sexuality and masculine reproductive health. Trabajo presentado en el Seminario Men, Family Formation and Reproduction, Buenos Aires, Argentina.

Infesta Domínguez, G. (2005). Las necesidades y requerimientos de los varones en materia de información y atención de la salud sexual y reproductiva. Buenos Aires: Universidad de Buenos Aires, Instituto de Investigaciones Gino Germán, Recuperado en agosto de 2011, de http://www.redaepa.org.ar/ jornadas/

viii/AEPA/B05/Infesta\%20Dominguez,\%20Graciela. pdf

Jiménez, E., Mosquera, M. \& Obregón, R. (2004). Comunicación, participación y salud sexual y reproductiva de los adolescentes reflexiones metodológicas desde la perspectiva de comunicación para el cambio social. Investigación y Desarrollo, 12(1), 78-107. 
Kelly, M. J. (2000). Planning for education in the context of HIV/AIDS (Fundamentals of educational planning № 66). París: UNESCO, International Institute for Educational Planning. Recuperado en agosto de 2011, de IIEP-UNESCO http://unesdoc.unesco. org/images/0012/001224/122405e.pdf

Lameiras, M., Rodríguez, Y., Calado, M. \& González, M. (2004). Creencias vinculadas al uso del preservativo masculino en adolescentes españoles sexualmente activos. Universitas Psychologica, 3(1), 27-34.

Lundgren, R. (2000, enero). Protocolos de investigación para el estudio de la salud sexual y reproductiva de los adolescentes varones y hombres jóvenes en América Latina. División de Promoción y Protección de la Salud, Programa de Familia y Población. Organización Panamerican de la Salud. Recuperado en agosto de 2011, de http://www.paho.org/Spanish/ HPP/HPF/ADOL/protocol.pdf

Madden, T. J., Scholder, E. P. \& Ajzen, I. (1992). A comparison of the theory of planned behavior and the theory of reasoned action. Personality and Social Psychology Bulletin, 18(1), 3-9.

Madiedo, J. (2001). Conducta sexual en adolescentes varones y anticoncepción. Revista Cubana de Enfermería, 17(1), 63-81.

Mosquera, M., Jiménez, E. \& Sanjuan, J. (2004). Evaluación y planificación de un plan de comunicación para la prevención de embarazos en adolescentes en el contexto escolar [Proyecto]. Barranquilla: Fundación Universidad del Norte.

Norris, A. \& Ford, K. (1995). Condom use by lowincome African American and Hispanic youth with a weil-known partner: Integrating the health belief model, theory of reasoned action, and the construct accesibility model. Journal of Applied Social Psychology, 25(20), 1801-1830.

Oblitas, L. A. (2004). Psicología de la salud y calidad de vida. México: Thomson.

Orgen, J. (2003). Some problems with social cognition models: A pragmatic and conceptual analysis. Health Psychology, 22(4), 424-428.

Pantelides, E. A. \& López, E. (2005). Varones latinoamericanos. Estudios sobre sexualidad y reproducción. Buenos Aires: Paidós.

Páramo, M. A. (2011). Factores de riesgo y factores de protección en la adolescencia: análisis de con- tenido a través de grupo de discusión. Terapia Psicológica, 29(1), 85-95.

Perdersen, W., Samuelsen, S. \& Wichstrom, L. (2003). Intercourse debut age: Poor resources, problem behavior or romantic appeal? The Journal of Sex Research, 40(4), 333-345.

Quiroz, N. \& Henríquez, A. (2009). Actitudes e información del varón relacionados a la utilización de métodos anticonceptivos [Versión electrónica]. Revista Salud, Sexualidad y Sociedad, 1(4). Disponible en http://www.inppares.org/revistasss/Libro\%204/ esta1.html

Ramiro, Maㅡ Teresa, Bermúdez, Maㅗ Paz, Sierra, Juan Carlos, \& Buela-Casal, Gualberto. (2013). Predicción del tipo de experiencia sexual en función de variables psicológicas y sociodemográficas en adolescentes panameños. Terapia psicológica, 31(2), 175-186.

Romer, D., Stanton, B., Galbraith, J., Feigelman, S., Black, M. M. \& Li, X. (1999). Parental influence on adolescent sexual behavior in high-poverty settings. Archives of Pediatric and Adolescent Medicine, 153(10), 1055-1062.

Sapién, J. \& Córdoba, D. (2011). Comportamiento sexual de varones durante el embarazo: casos en la ciudad de México. Terapia Psicológica, 29(2), 185-190.

Soravisutr, C. (2002). La educación para la prevención del VIH/SIDA. Perspectivas: Revista Trimestral de Educación Comparada, 32(2), 78-85.

Valdivia, C. \& Vizcarra, B. (en prensa). Consumo de drogas y violencia en el noviazgo en jóvenes universitarios del sur de Chile. Terapia Psicológica.

Vargas-Trujillo, E. \& Barrera, F. (2003). Actividad sexual y relaciones románticas durante la adolescencia: Algunos factores explicativos (Documentos CESO № 56). Bogotá: Universidad de los Andes.

Vargas-Trujillo, E., Barrera, F., Burgos, M. \& Daza, B. (2006). La intención de los jóvenes de tener relaciones sexuales en la adolescencia: papel de la televisión, la relación con los padres y las cogniciones. Universitas psychologica, 5(1), 69-84.

Vega, J. (2009). Caracterización de las normas sociales, actitudes, habilidades para la vida, intenciones y comportamientos relacionados con la posibilidad de ocurrencia de embarazos en adolescentes. Estudio 
Ana Mercedes Bello-Villanueva, Jorge Palacio, Pablo Vera-Villarroel, Oscar Oviedo-Trespalacios, Melissa Rodríguez-Diaz, Karen Celis-Atenas, Paula Pavez.

de línea base cuantitativa y cualitativa (Informe de resultados: línea de base población adolescente de 12-20 años). Barranquilla: Fundación Universidad del Norte.

Whitaker, D., Miller, K. \& Clark, L. (2000). Reconceptualizing adolescents sexual behavior: Beyond did they or didn't they? Family Planning Perspectives, 32(3), 111-117.

Zárate, O. \& Calderón, V. (2010). Elaboración de una escala de actitudes respecto a la sexualidad de personas con discapacidad mental. Revista Electrónica de Psicología Iztacala, 13(4), 106-115. 